\title{
Focus classification in digital holographic microscopy using deep convolutional neural networks
}

\author{
Tomi Pitkäaho $^{a}$, Aki Manninen ${ }^{b}$ and Thomas J. Naughton ${ }^{a}$ \\ ${ }^{a}$ Department of Computer Science, Maynooth University-National University of Ireland Maynooth, Maynooth, County \\ Kildare, Ireland

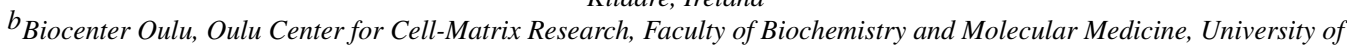 \\ Oulu, Finland
}

\begin{abstract}
In digital holographic microscopy, one often obtains an in-focus image of the sample by applying a focus metric to a stack of numerical reconstructions. We present an alternative approach using a deep convolutional neural network.
\end{abstract}

OCIS codes: $090.1995,100.6890$

\section{Introduction}

Digital holographic microscopy (DHM) is a label-free, single-shot technique that is well suited for imaging three dimensional objects [1]. DHM overcomes a problem present in conventional optical microscopes of a shallow depth-of-field, permitting one to reconstruct at different in-focus planes of a volume. Nevertheless, an object of interest is usually in-focus only in one or few depths as a single reconstruction layer still has a shallow depth-of-field. To solve the problem of finding a focus plane of an object different methods have been proposed. These methods can be based on self-entropy [2], amplitude analysis [3], power spectra [4] or other metrics [5]. Common to all of these single wavelength methods is that they reconstruct a stack of images that the focus metric is applied to. A minimum or a maximum value, depending on the used focus metric, is used to determine the in-focus plane. This procedure is applied to each of the holograms. We propose to use deep learning as an autofocusing method. The greatest benefit of the proposed method is that after the training is completed, the in-focus plane can be obtained by using only the single in the hologram plane and without any reconstruction.

\section{Digital holographic microscopy}

A magnified digital hologram $H_{0}(x, y)=|R|^{2}+|O|^{2}+R^{*} O+R O^{*}$ that is sampled by a digital camera, can be propagated at any depth $z$ of the reconstruction volume using the Fresnel approximation [6] as

$$
\begin{aligned}
U(x, y ; z) & = \\
& \frac{-i}{\lambda z} \exp (\mathrm{i} k z) I_{0}(x, y) \otimes \exp \left[\mathrm{i} \pi \frac{x^{2}+y^{2}}{\lambda z}\right]
\end{aligned}
$$

where $\lambda$ is the wavelength of the light, $\otimes$ denotes a convolution operation, and $k=2 \pi / \lambda$. The terms $R^{*}$ and $O^{*}$ denote the complex conjugates of the reference wave and the object wave, respectively. From the complex valued reconstruction, the amplitude component is defined

$$
I(x, y ; z)=\sqrt{\operatorname{Re}[U(x, y ; z)]^{2}+\operatorname{Im}[U(x, y ; z)]^{2}}
$$

\section{Deep learning}

Deep learning is a computational approach that enables multiple levels of abstraction and can be used efficiently in various applications [7]. In practice, every neural network with more than one hidden layer can be considered as a deep learning architecture. A convolutional neural network has one or more layers that perform convolution operations on its two-dimensional input. Deep convolutional neural networks that are one form of deep learning have been used successfully in various different visual object recognition and object detection applications [8-10].

Advances in Microscopic Imaging, edited by Emmanuel Beaurepaire, Francesco Saverio Pavone,

Peter T. C. So, Proc. of SPIE-OSA Vol. 10414, 104140K · C 2017 OSA-SPIE

CCC code: $1605-7422 / 17 / \$ 18 \cdot$ doi: $10.1117 / 12.2286161$ 
We chose a convolutional neural network approach to tackle the autofocusing problem still existing in digital holographic microscopy of transparent samples. The architecture of the network is based on the AlexNet architecture that won the Large Scale Visual Recognition Challenge 2012 [9].

\subsection{Training}

In total 494 holograms of semitransparent Madine Darby canine kidney (MDCK) multicellular samples were prepared and captured by using an off-axis Mach Zehnder digital holographic microscope (Lyncée Tec T1000, Lyncée Tec SA, Lausanne, Switzerland) with $660 \mathrm{~nm}$ laser source and 40X microscope objective. Each hologram was numerically reconstructed at the middle region of a sample by using a manually chosen reconstruction depth. Each hologram was reconstructed to 20 other depths ( $\pm 100 \mathrm{~mm}$ from the in-focus plane) with the reconstruction step being $10 \mathrm{~mm}$. The data was processed and augmented by scaling each amplitude reconstruction down to $256 \times 256$ pixel image, that was followed by five $227 \times 227$ cropping operations to each corner and the center. Each cropped image was rotated three times ( 90 degree rotations) and each resulting image was augmented through horizontal mirroring. In addition each $1024 \times 1024$ pixel amplitude reconstruction was scaled down to $227 \times 227$ pixel image that underwent the same rotation and mirroring augmentation procedures. After data processing and augmentation the total amount of images used in training was 485856 , which was divided to actual training data $(90 \%, 437271)$ and validation data $(10 \%, 48585)$. Example images used in the training are shown in the Fig. 1. The learning rate was set to 0.001. During the training a mean pixel value over the whole training set was subtracted from each training image pixel.

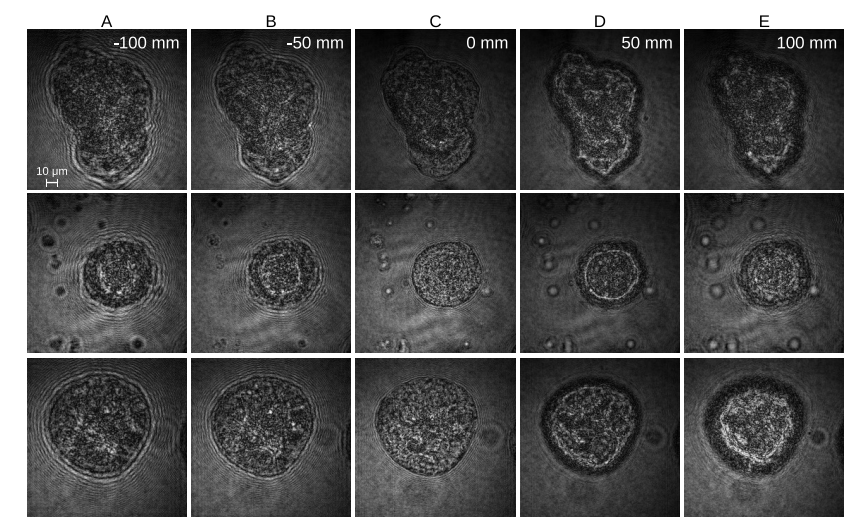

Fig. 1. $227 \times 227$ pixel example images used in training. Each image is an amplitude reconstruction of a hologram. Column A $-100 \mathrm{~mm}$, column B $-50 \mathrm{~mm}$, column C $0 \mathrm{~mm}$, column D $50 \mathrm{~mm}$, and column E $100 \mathrm{~mm}$ from the in-focus plane, respectively.

\section{Results}

The network was tested with 12 holograms that went through the same preprocessing as the images used in the training (including the augmentation). In total, 12096 images were used in the testing. A histogram of the test result is shown in the Fig. 2. In addition, the method was tested by using amplitude images of the hologram plane. Fig. 3 shows example amplitude images on the hologram plane.

\section{Conclusions}

In this paper, it was shown that autofocusing of digital holograms of semitransparent biological samples can be achieved efficiently by using deep learning. The method was theoretically described and experimental results were demonstrated.

\section{Acknowledgements}

This research was supported by an Irish Research Council Postgraduate Scholarship, Science Foundation Ireland under Grant No. 13/CDA/2224, and Kerttu Saalasti Foundation. 


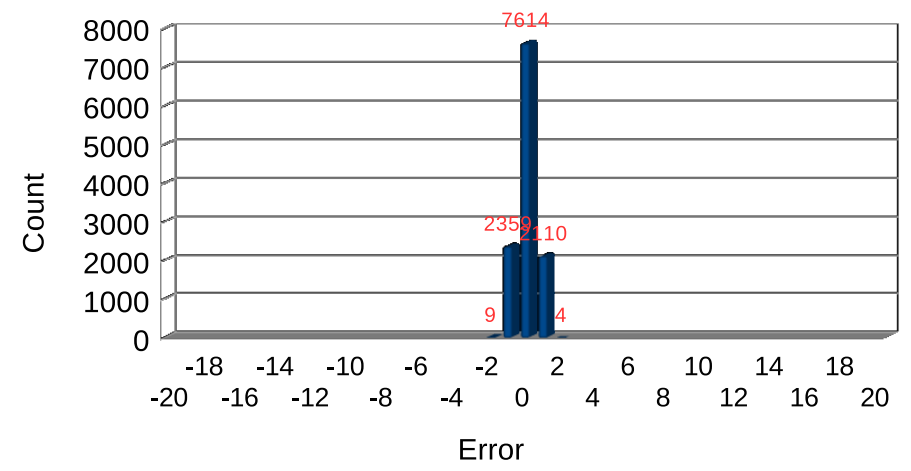

Fig. 2. Histogram of the testing phase. Showing in-focus depth estimate errors between the groundtruth and an estimate returned by the trained convolutional neural network. $99.9 \%$ of the estimates are within one reconstruction step.
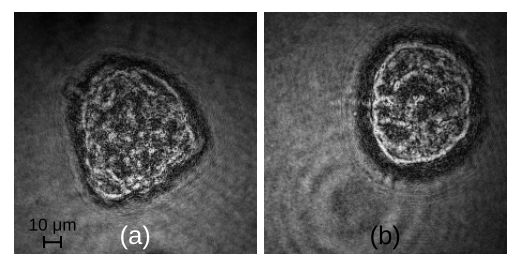

Fig. 3. $227 \times 227$ pixel example images used in the testing. An average absolute error in in-focus depth estimate returned by the convolutional neural network was $5.1 \mathrm{~mm}$ by using 12 test holograms.

\section{References}

1. E. Cuche, F. Bevilacqua, and C. Depeursinge, "Digital holography for quantitative phase-contrast imaging," Optics Letters 24, 291-293 (1999).

2. J. Gillespie and R. A. King, "The use of self-entropy as a focus measure in digital holography," Pattern Recognition Letters 9, 19-25 (1989).

3. F. Dubois, C. Schockaert, N. Callens, and C. Yourassowsky, "Focus plane detection criteria in digital holography microscopy by amplitude analysis," Optics Express 14, 5895-5908 (2006).

4. P. Langehanenberg, G. von Bally, and B. Kemper, "Autofocusing in digital holographic microscopy," 3D Research 2, 1 (2011).

5. I. Bergoënd, T. Colomb, N. Pavillon, Y. Emery, and C. Depeursinge, "Depth-of-field extension and 3D reconstruction in digital holographic microscopy," Proc. SPIE 7390, 73901C-1 (2009).

6. J. Goodman, Introduction to Fourier Optics, Roberts and Company (2005).

7. Y. LeCun, Y. Bengio, and G. Hinton, "Deep learning," Nature 521, 436-444 (2015).

8. Y. LeCun, B. Boser, J. S. Denker, D. Henderson, R. E. Howard, W. Hubbard, L. D. Jackel, "Handwritten digit recognition with a back-propagation network," In Advances in Neural Information Processing Systems, 396-404 (1990).

9. A. Krizhevsky, I. Sutskever, and G. E. Hinton, "Imagenet classification with deep convolutional neural networks," In Advances in Neural Information Processing Systems, 1097-1105 (2012).

10. K. He, X. Zhang, S. Ren, and J. Sun, "Deep residual learning for image recognition," arXiv preprint arXiv:1512.03385, (2015). 